\title{
Safety and Efficacy of High Versus Standard Starting Doses of Insulin Glargine in Overweight and Obese Chinese Individuals with Type 2 Diabetes Mellitus Inadequately Controlled on Oral Antidiabetic Medications (Beyond VII): Study Protocol for a Randomized Controlled Trial
}

Linong Ji · Zhengnan Gao · Bimin Shi · Rongwen Bian •

Fuzai Yin · Wuyan Pang · Hong Gao · Nan Cui

Received: March 15, 2018 / Published online: June 5, 2018

(C) The Author(s) 2018

\section{ABSTRACT}

Background: Treatment with basal insulin in Chinese populations is currently sub-optimal, with delayed initiation of insulin treatment and inadequate dose titration. Increasing the initial dose of insulin may be a practicable and effective solution to the problem of titration. A higher initial dose will be helpful for patients to achieve the blood glucose target and improve

Enhanced Digital Features To view enhanced digital features for this article go to https://doi.org/10.6084/ m9.figshare.6269984.

L. Ji $(\bowtie)$

Department of Endocrinology and Metabolism, Peking University People's Hospital, 11 Xizhimen South Street, Xicheng District, Beijing 100044, China

e-mail: jiln@bjmu.edu.cn

Z. Gao

Department of Endocrinology and Metabolism, Dalian Municipal Central Hospital Affiliated of Dalian Medical University, 826 Xi'nan Road, Dalian 116033, Liaoning, China

B. Shi

Department of Endocrinology and Metabolism, The First Affiliated Hospital of Soochow University, 188 Shizi Street, Suzhou 215006, Jiangsu, China

R. Bian

Department of Endocrinology and Metabolism, Jiangsu Province Institute of Geriatrics, 30 Luojia Road, Nanjing 210024, Jiangsu, China treatment satisfaction and compliance as well require fewer steps to titrate. Considering that overweight and obese patients usually require higher insulin doses because of insulin resistance, a higher initial dose of the basal insulin is feasible in overweight and obese patients with type 2 diabetes. However, safety is an important issue needing to be considered for higher initial dose treatment. The aim of this study is to assess the safety and efficacy of higher $(0.3 \mathrm{U} / \mathrm{kg})$ compared with standard $(0.2 \mathrm{U} / \mathrm{kg})$ starting doses of basal insulin in overweight and obese Chinese patients with type 2 diabetes who have failed to achieve glycaemic control using oral antidiabetic drugs (OADs).

F. Yin

Department of Endocrinology and Metabolism, The First Hospital of Qinhuangdao, 258 Wenhua Road, Qinhuangdao 066000, Hebei, China

W. Pang

Department of Endocrinology and Metabolism, Huaihe Hospital of Henan University, 115 Ximen Street, Kaifeng 475000, Henan, China

H. Gao · N. Cui

Sanofi China, 19 Floor, Tower III, Jing'An Kerry Centre, 1228 Middle Yan'an Road, Shanghai 200040, China 
Methods: This is a phase IV, randomized, noninferiority, open-label trial that will be conducted at approximately 50 centers in China. Eight hundred eighty overweight and obese adult Chinese patients with type 2 diabetes will be randomized to receive higher $(0.3 \mathrm{U} / \mathrm{kg})$ or standard $(0.2 \mathrm{U} / \mathrm{kg})$ starting doses of basal insulin glargine $(100 \mathrm{U} / \mathrm{ml})$ during a 16-week period. The primary endpoint is whether a higher initial dose of basal insulin $(0.3 \mathrm{U} / \mathrm{kg})$ is non-inferior to a standard initial dose $(0.2 \mathrm{U} / \mathrm{kg})$ based on the percentage of patients with at least one episode of hypoglycaemia $(\leq 3.9 \mathrm{mmol} / \mathrm{l}$ or severe) over 16 weeks. Secondary endpoints include evaluation of glycosylated haemoglobin A1c (HbA1c), fasting blood glucose, postprandial blood glucose, insulin dose and safety.

Discussion: This study is the first randomizedcontrolled study to evaluate the safety and efficacy of basal insulin treatment with a higher starting dose versus standard starting dose in overweight and obese Chinese patients with type 2 diabetes. Results of this study could generate evidence to support the feasibility of a higher starting dose of basal insulin in diabetes management of overweight and obese Chinese patients, therefore providing an easy approach to improve diabetes management.

Trial Registration: ClinicalTrials.gov identifier, NCT02836704. Registered on July 7th 2016.

Funding: Sanofi China.

Keywords: Basal insulin; BMI; Insulin glargine; Obesity; Type 2 diabetes

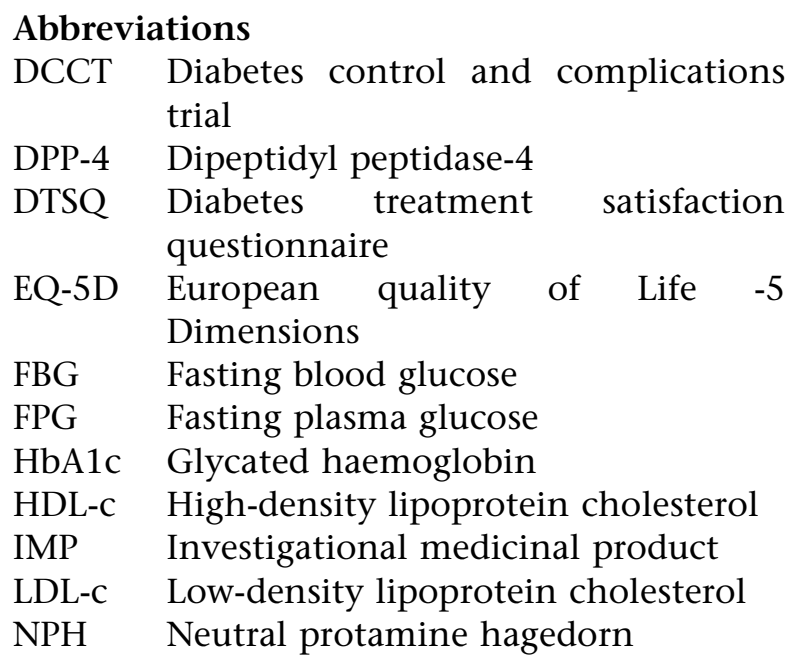

OAD Oral antidiabetic drug

ORBIT Observational Registry of Basal Insulin Treatment

PPG Postprandial plasma glucose

SAE Serious adverse event

SMBG Self-monitored blood glucose

SPIRIT Standard protocol items for clinical trials

T2DM Type 2 diabetes mellitus

TC Total cholesterol

TG Triglyceride

UKPDS UK Prospective Diabetes Study

\section{INTRODUCTION}

The incidence of type 2 diabetes mellitus (T2DM) in China has increased rapidly over the past 2 decades [1, 2]. By 2040, an estimated 642 million people worldwide will live with diabetes and 150.7 million are in China [3]. A study reported by the International Diabetes Federation (IDF) and the Chinese Diabetes Society (CDS) in 2010 found that $13 \%$ of all medical expenditures in China (US\$ 25 billion per annum) was directly caused by diabetes [4]. Therefore, unless improvements are made in both the prevention and treatment of diabetes in China, the economic and healthcare burden will continue to rise.

One way to reduce the healthcare and economic burden of diabetes patients is to achieve and maintain good glycaemic control, thus reducing the risk of diabetes-related complications. Like the American Diabetes Association standards of medical care in diabetes [5], Current Chinese treatment guidelines [6] recommend a stepwise approach to diabetes management, with patients initially receiving lifestyle intervention and therapy with oral antidiabetic drugs (OADs). As T2DM is a progressive disease, with a gradual decrease in the number of functional insulin-producing $\beta$-cells, patients often require basal insulin treatment if glycaemic control cannot be maintained by OADs.

Insulin glargine is a long-acting basal insulin analogue that is widely used in the glycaemic control of type 2 diabetes patients [7, 8]. 
Adequate starting doses of insulin and an appropriate titration algorithm are important to achieve optimal glycaemic control. The treat to target trial demonstrated that titrating the insulin glargine dose using a fasting plasma glucose (FPG) target of $\leq 6.1 \mathrm{mmol} / \mathrm{l}$ resulted in good glycaemic control $(58 \%$ of individuals achieved HbA1c $<7 \%$ ) and a low incidence of nocturnal hypoglycaemia [9].

Although a number of clinical studies have demonstrated the benefits of sufficient insulin dosing on glycaemic management, the current practice in China remains sub-optimal in both the time to initiation of insulin treatment and the subsequent titration of insulin in patients with inadequate glycaemic control. A study of almost 240,000 patients with T2DM throughout China found that among those treated with OADs and/or insulin, less than $1 / 3$ of patients achieved the glycaemic target of HbA1c $<7 \%$ [10]. Similarly, the First Basal Insulin Evaluation (FINE) study assessed real-world insulin practices in T2DM patients from 2006 to 2008 across 11 countries in Asia and found that initiation of insulin therapy was delayed by approximately 9 years in these patients, despite their high HbA1c levels [11]. Furthermore, there was an overall lack of insulin titration in Asian patients with the average insulin dose only increasing by approximately $0.03 \mathrm{U} / \mathrm{kg}$ after 6 months' treatment and only $33.7 \%$ of patients achieved their HbA1c targets [11]. A more recent Observational Registry of Basal Insulin Treatment (ORBIT) study, conducted from 2011 to 2013 across 209 hospitals in China, found that more than onethird of patients with uncontrolled diabetes at follow-up visits did not receive insulin titration, and the average increase in basal insulin dose at 6 months was only $0.03 \mathrm{U} / \mathrm{kg}$. The ORBIT investigators suggested that Chinese type 2 diabetes patients tend to have a delayed insulin initiation and efforts to improve insulin utilization are urgently needed to achieve and maintain glycaemic control [8]. Similar challenges with sub-optimal insulin titration in realworld practice have been demonstrated in other Asian countries. In a sub-analysis of the Add-on Lantus to Oral Hypoglycemic Agents (ALOHA) study, Odawara et al. found that an appropriate starting dose was essential to achieving target
HbA1c in insulin-naïve Japanese diabetes patients. Furthermore, patients who did not receive insulin titration had a significantly lower chance of achieving HbA1c targets [12].

Considering the current healthcare system barriers in China, inadequate glucose control will continue to exist for a long time and the challenge of insulin titration in Chinese diabetes patients requires long-term patient education. It has been suggested that initiating insulin at higher starting doses may be of benefit. Inadequate titration of insulin can lead to an insufficient total dose of insulin, which may cause sub-optimal glycaemic control and a low rate of patients achieving glycaemic control targets. Therefore, initiating treatment with a higher dose of insulin may represent a treatment approach that will partially compensate for the current insufficiency in dose titration. However, hypoglycaemia is another concern for most physicians and patients when they are considering using a higher initial dose of insulin. Currently, standard basal insulin is recommended by the American Diabetes Association (ADA) and American Association of Clinical Endocrinologists (AACE), starting with $0.1-0.2 \mathrm{U} / \mathrm{kg}$ per day depending on the degree of hyperglycaemia $[5,13]$. In China, the recommended standard starting dose is $0.2 \mathrm{U} / \mathrm{kg}$ per day [6].

The AACE guidelines recommend a higher initial dose of basal insulin $(0.2-0.3 \mathrm{U} / \mathrm{kg})$ for diabetes patients with $\mathrm{HbA1c}>8 \%$ [13]. In addition, considering the impact of body weight on insulin resistance, a BMI-based insulin dose regimen has recently been described [14]; the BMI-based higher dose of initial insulin demonstrated equal safety efficacy and safety compared with a standard starting dose of insulin. Furthermore, several interventional studies conducted in China have shown that the final insulin dosing after 16 weeks of treatment is as high as $0.3-0.5 \mathrm{U} / \mathrm{kg}$ in order to achieve glycaemic control $[15,16]$. Taking into account these factors, this study will initiate insulin therapy at a dose of $0.3 \mathrm{U} / \mathrm{kg}$ in overweight and obese Chinese diabetes patients with inadequate glycaemic control on OADs.

Few data are available regarding the use of a higher starting dose of insulin, especially 
regarding potential hypoglycaemia risk. This is the first study that will be conducted in Chinese diabetes patients that investigates the safety of initiating insulin treatment at a higher dose. It will enroll patients who are overweight or obese as these patients will most likely require a higher dose of insulin. This study will therefore assess the safety and efficacy of a higher starting dose of $0.3 \mathrm{U} / \mathrm{kg}$ basal insulin compared with the standard starting dose of $0.2 \mathrm{U} / \mathrm{kg}$ in overweight or obese diabetes patients in China. The study will also assess whether a higher starting dose has a positive effect on current sub-optimal titration practices and insufficient insulin dosage in China.

\section{METHODS AND DESIGN}

\section{Setting}

This is a phase IV, randomized, non-inferiority, open-label trial that will be conducted in 880 diabetes patients at approximately 50 study centers in China. The aim is to assess the safety and efficacy of a higher $(0.3 \mathrm{U} / \mathrm{kg})$ compared with standard $(0.2 \mathrm{U} / \mathrm{kg})$ starting dose of basal insulin in overweight and obese Chinese patients with type T2DM who have failed to achieve glycaemic control using OADs. The standard starting dose of $0.2 \mathrm{U} / \mathrm{Kg}$ was chosen as this is the current recommended initial dose of basal insulin $[11,17]$. The comparator starting dose of $0.3 \mathrm{U} / \mathrm{kg}$ was chosen on the basis of patients' characteristics of uncontrolled blood glucose levels [13], the previous research experience of the BMI-based starting dose of insulin glargine in non-Chinese populations [14] and data from Chinese studies indicating that the final dose of insulin required to achieve glycaemic control can be $0.3-0.5 \mathrm{U} / \mathrm{kg}$ after 16 weeks of treatment $[15,16]$.

All procedures performed in studies involving human participants will be in accordance with the ethical standards of the institutional and/or national research committee and with the 1964 Helsinki Declaration, as revised in 2013. Informed consent will be obtained from all individual participants included in the studies.

\section{Study Objectives}

The primary objective is to determine whether a higher initial dose of basal insulin glargine $(0.3 \mathrm{U} / \mathrm{kg})$ is non-inferior to a standard initial dose $(0.2 \mathrm{U} / \mathrm{kg})$ based on the percentage of patients with at least one episode of hypoglycaemia ( $\leq 3.9 \mathrm{mmol} / \mathrm{l}$ or severe) during the 16 weeks of treatment.

Secondary study objectives include evaluation of the percentage of patients achieving $\mathrm{HbA} 1 \mathrm{c}$ $<7 \%$ at week 16 and the percentage and accumulated percentage of patients achieving FPG targets $(<5.6,<6.1$ and $<7.0 \mathrm{mmol} / \mathrm{l})$ from baseline to week 16. Changes in HbA1c, FPG, postprandial plasma glucose (PPG) and insulin dose (units per day and U/kg) from baseline to week 16 will also be evaluated. The safety profile will be assessed, including adverse events and the incidences of overall hypoglycaemia, nocturnal hypoglycaemia and severe hypoglycaemia throughout the treatment period. Patient and physician satisfaction and adherence will also be assessed, including the drop-out rate and percentage of patients able to comply with insulin treatment and titration.

A planned sub-group analysis will evaluate the safety and efficacy (including the glycaemic control rate and changes in HbA1c, FPG and PPG), stratified by patient age, duration of T2DM, baseline OAD treatment and baseline levels of HbA1c, FPG and PPG.

\section{Patients}

Participants aged between 18 and 70 years with a confirmed diagnosis of T2DM for at least 2 years who have received continuous treatment with two to three OADs (including metformin $\geq 1.5 \mathrm{~g} /$ day or at the maximum tolerated dose) for $>3$ months and have an HbA1c level between $7.5 \%$ and $11 \%$ with fasting blood glucose (FBG) level of $>9 \mathrm{mmol} / \mathrm{l}$ and BMI between 25 and $40 \mathrm{~kg} / \mathrm{m}^{2}$ are eligible for this study. Moreover, patients must agree to perform self-monitoring of blood glucose (SMBG) using the sponsor-provided blood glucose meter. Patients with diabetes other than confirmed type 2 diabetes are excluded. In 
addition, patients are excluded if they are treated with insulin or thiazolidinediones or GLP-1 receptors agonists during the 3 months prior to screening, received pharmacological treatments for weight loss during the 3 months prior to screening, have a history of diabetic ketoacidosis or hyperosmolar nonketotic coma, have a history of hypoglycaemia unawareness or unexplained hypoglycaemia in the past 6 months, are pregnant or breast feeding, or are not using an acceptable method of birth control.

\section{Study Design}

\section{Screening Period}

Following written informed consent, patients will enter a 2-week screening phase (Fig. 1). During this phase, the patient will continue on their current stable doses of OADs and lifestyle interventions.

\section{Run-In Period}

At the end of the screening period, patients will enter a 3-day run-in period. During this run-in period, 3 days prior to randomization, patients will cease sulfonylurea or glinide treatment, but continue other OAD agents at the same dosage and frequency. If the DPP-4 inhibitor used has not obtained approval for being combined with insulin, it must be stopped at randomization. Patient will be trained in the use of the blood glucose meter and insulin injections. During the run-in period, if a patient has FBG levels $>16.7 \mathrm{mmol} / \mathrm{l}$, which is later confirmed by the fasting plasma glucose (FPG), they should discontinue the run-in process, should be appropriately treated in accordance with routine clinical practice and are therefore excluded from the study.

\section{Randomization}

Following successful completion of the run-in period, the patient is randomized to receive either a standard dose or higher dose basal insulin injection. Randomization is based on a computer-generated code stratified by baseline sulfonylurea or glinide use, and allocation is performed via a centralized interactive voice response system or interactive web response system. Patients are randomized in a 1:1 ratio to receive either a standard initial dose $(0.2 \mathrm{U} / \mathrm{kg})$ or a higher initial dose $(0.3 \mathrm{U} / \mathrm{kg})$ of insulin glargine (100 U/ml, Lantus ${ }^{\circledR}$, Sanofi).

\section{Treatment Period}

The study medication is provided as a pre-filled Lantus SoloSTAR insulin pen containing $3 \mathrm{ml}$ of

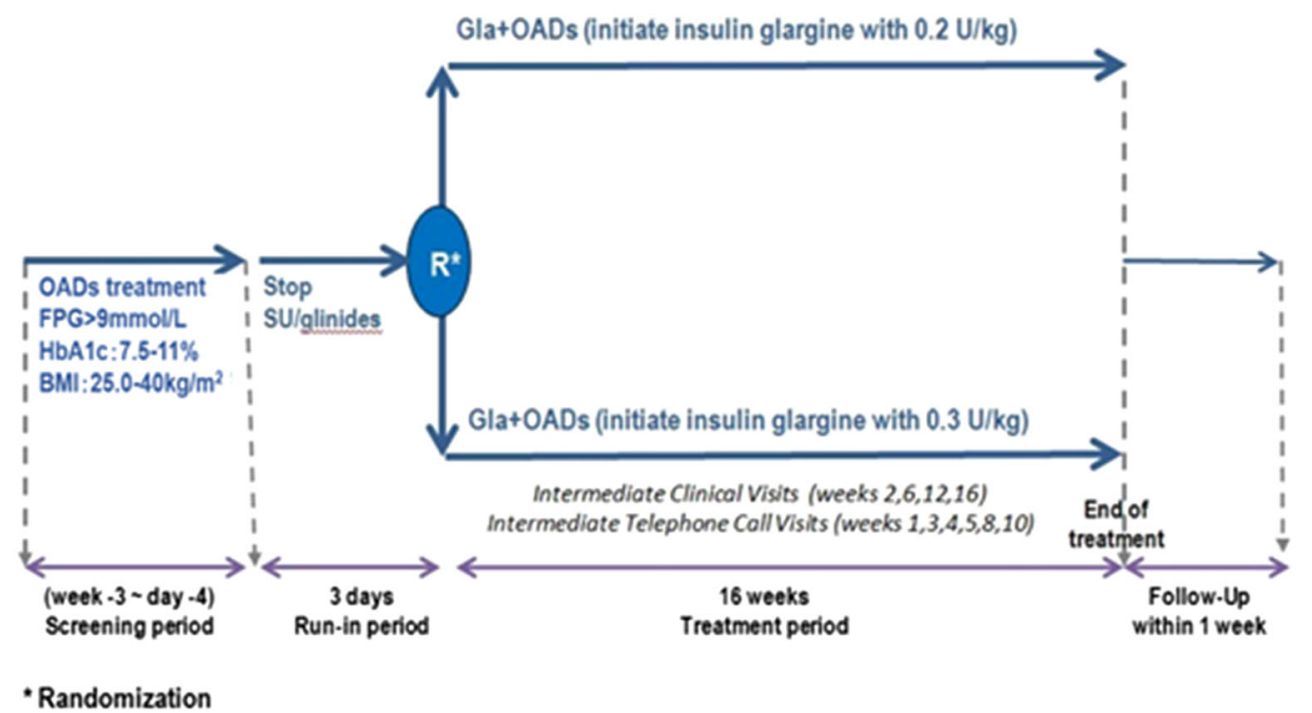

Fig. 1 Study design algorithm. FPG fasting blood glucose; BMI body mass index; Gla insulin glargine; $O A D$ oral antidiabetic drug; $S U$ sulfonylurea 
$100 \mathrm{U} / \mathrm{ml}$ insulin glargine. After randomization to either standard or higher initial doses, patients will be injected once a day at the same time, usually at bedtime, for a period of 16 weeks. The starting daily dose will be $0.2 \mathrm{U} /$ $\mathrm{kg}$ of body weight (standard initial dose arm) or $0.3 \mathrm{U} / \mathrm{kg}$ of body weight (higher initial dose arm). A forced dose titration will be conducted at each subsequent visit to keep the FBG level between $\quad 4.4 \mathrm{mmol} / \mathrm{l}$ and $5.6 \mathrm{mmol} / \mathrm{l}$ (80-100 mg/dl). Titration will be based on the FBG levels of 3 consecutive days (see insulin titration).

During this treatment period, patients in both arms will continue their non-sulfonylurea and non-glinide OAD therapy at the same dosage and frequency prior to study enrolment. Patients may withdraw from treatment with the medication if they decide to do so, at any time and for any reason, or this may be the investigator's decision.

\section{Self-Monitored Blood Glucose Test (SMBG)}

The 4-point (before breakfast and $2 \mathrm{~h}$ after the start of breakfast, lunch and dinner) SMBG should be performed and recorded by patients on their own at the last day of the run-in period and at least 3 consecutive days within the week prior to visit 13 (week 16) or in case of premature discontinuation. During the treatment period, SMBG, at least for FBG, should be performed and recorded at least on 3 consecutive days within the week prior to each visit. Meanwhile, all SMBG values whenever the patient experiences symptoms possibly related to hypoglycaemia will also be recorded.

\section{Insulin Titration}

The titration will be done at each visit by a physician. A forced dose titration will be conducted at each subsequent visit to keep the FBG level between $4.4 \mathrm{mmol} / \mathrm{l}$ and $5.6 \mathrm{mmol} / \mathrm{l}$ $(80-100 \mathrm{mg} / \mathrm{dl})$. The physician will look at the FBG values of the last 3 consecutive days to adjust insulin doses. The FBG value used for the titration is the median of the three values, except if one is below $4.4 \mathrm{mmol} / \mathrm{l}$; in this case, this lowest value will be used (Table 1). If there is one FBG value missing (only two available),
Table 1 Titration algorithm for insulin glargine

\begin{tabular}{ll}
\hline $\begin{array}{l}\text { Median fasting blood } \\
\text { glucose }(\mathbf{F P G}), \mathbf{m m o l} / \mathbf{1}\end{array}$ & $\begin{array}{l}\text { Insulin glargine dose } \\
\text { adjustment, units }\end{array}$ \\
\hline$\geq 5.6$ and $<6.7$ & $\begin{array}{l}0-2 \text { (at the discretion of the } \\
\text { investigator/physician) }\end{array}$ \\
$\geq 6.7$ and $<7.8$ & 2 \\
$\geq 7.8$ and $<10.0$ & 4 \\
$\geq 10$ & 6 \\
$<4.4$ & -2 \\
\hline
\end{tabular}

dose titration should be according to the lower value. If there are two FBG values missing (only one available), the dose adjustment should be postponed to the next visit.

\section{Definitions of Hypoglycaemia}

Hypoglycaemia is categorized as follows: confirmed hypoglycaemia is defined as a measured blood glucose level $\leq 3.9 \mathrm{mmol} / \mathrm{l}$ with or without clinical symptoms of hypoglycaemia; nocturnal hypoglycaemia is defined as hypoglycaemia (blood glucose level $\leq 3.9 \mathrm{mmol} / \mathrm{l}$ ) that occurs during sleep (0:00-5:59 a.m.) and responds to ingestion of carbohydrates; severe hypoglycaemia is defined as an event with clinical symptoms resulting from hypoglycaemia in which the study subject requires assistance for the administration of carbohydrate, glucagon or other treatments and a measured blood glucose level $<2 \mathrm{mmol} / \mathrm{l}$ or prompt recovery following oral carbohydrate, intravenous glucose or glucagon administration.

\section{Data Collection}

Participants have mandatory clinic visits during the screening, run-in, randomization periods and then at weeks $2,6,12$ and 16 post-randomization during the treatment period (Fig. 2). Telephone follow-up is conducted at weeks $1,3,4,5,8$ and 10 post-randomization and 1 week after the end of the study treatment schedule. FBG levels are assessed at run-in and weeks $2,6,12$ and 16 post-randomization. 


\begin{tabular}{|c|c|c|c|c|c|c|c|c|c|c|c|c|c|c|}
\hline \multirow[b]{2}{*}{ Week( \pm day $) \#$} & \multirow{2}{*}{\begin{tabular}{|c|}
$\begin{array}{c}\text { ccreening } \\
\text { Period }\end{array}$ \\
$-3 w \sim-4 d$ \\
\end{tabular}} & \multirow{2}{*}{ 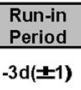 } & \multirow{2}{*}{\begin{tabular}{|c|} 
Baseline \\
0
\end{tabular}} & \multicolumn{10}{|c|}{ Treatment Period } & \multirow{2}{*}{$\begin{array}{l}\begin{array}{l}\text { End of } \\
\text { Study }\end{array} \\
16 \sim 17\end{array}$} \\
\hline & & & & $1( \pm 3)$ & $2( \pm 3)$ & $3( \pm 3)$ & $4( \pm 3)$ & $5( \pm 3)$ & $6( \pm 3)$ & $8( \pm 3)$ & $10( \pm 3)$ & $12( \pm 3)$ & $16( \pm 3)$ & \\
\hline Visit \# & 1 & 2 & 3 & 4 & 5 & 6 & 7 & 8 & 9 & 10 & 11 & 12 & 13 & 14 \\
\hline Type of contact & Visit & Visit & Visit & $\begin{array}{c}\text { Phone } \\
\text { call }\end{array}$ & Visit & $\begin{array}{c}\text { Phone } \\
\text { call }\end{array}$ & $\begin{array}{c}\text { Phone } \\
\text { call }\end{array}$ & $\begin{array}{l}\text { Phone } \\
\text { call }\end{array}$ & Visit & $\begin{array}{c}\text { Phone } \\
\text { call }\end{array}$ & $\begin{array}{c}\text { Phone } \\
\text { call }\end{array}$ & Visit & Visit & Phone call \\
\hline $\begin{array}{l}\text { Inclusion and Clinical evaluation } \\
\text { Informed consent }\end{array}$ & & & & & & & & & & & & & & \\
\hline $\begin{array}{l}\text { Demography } \\
\end{array}$ & $\frac{x}{x}$ & & & & & & & & & & & & & \\
\hline Medical and surgical history & $\mathrm{x}$ & & & & & & & & & & & & & \\
\hline Previous and concomitant medication & $\mathrm{x}$ & $x$ & $\mathrm{x}$ & $x$ & $\mathrm{x}$ & $x$ & $x$ & $x$ & $x$ & $\mathrm{x}$ & $x$ & $x$ & $x$ & $x$ \\
\hline Physical examination & $\mathrm{X}$ & & & & & & & & & & & & $x$ & \\
\hline Vital signs & $\mathrm{X}$ & - & $x$ & & & & & & & & & & $\mathrm{X}$ & \\
\hline Body Weight & $\frac{x}{x}$ & & $\mathrm{x}$ & & & & & & & & & & $x$ & \\
\hline Height & $\mathrm{x}$ & & & & & & & & & & & & & \\
\hline Inclusion/exclusion criteria & $\mathrm{x}$ & $\mathrm{x}$ & $\frac{\mathrm{x}}{\mathrm{x}}$ & & & & & & & & & & & \\
\hline Randomization & & & $\mathrm{x}$ & & & & & & & & & & & \\
\hline HbA1C & & $x^{*}$ & $x$ & & & & & & & & & & $x$ & \\
\hline $\begin{array}{l}\text { Lipid profile (TC, HDL-c, LDL-c, TG) in } \\
\text { fasting state }\end{array}$ & & $x^{*}$ & & & & & & & & & & & $\mathrm{x}$ & \\
\hline $\begin{array}{l}\text { Biochemistry (Serum creatinine, ALT, } \\
\text { AST, total bilirubin) }\end{array}$ & & $x^{*}$ & & & & & & & & & & & $\mathrm{x}$ & \\
\hline Biochemistry (FPG) & & $x^{*}$ & $x$ & & $\mathrm{x}$ & & & & $\mathrm{x}$ & & & $\mathrm{x}$ & $\mathrm{x}$ & \\
\hline $\begin{array}{l}\text { Serum pregnancy test (females with } \\
\text { child bearing potential only) }\end{array}$ & & $x^{*}$ & & & & & & & & & & & $\mathrm{x}$ & \\
\hline Ireatment & & & & & & & & & & & & & & \\
\hline $\begin{array}{l}\text { discontinue the treatment of } \\
\text { sulfonylurea or glinides }\end{array}$ & & $x$ & $x$ & $\mathrm{x}$ & $\mathrm{x}$ & $\mathrm{x}$ & $x$ & $x$ & $x$ & $x$ & $x$ & $x$ & $x$ & \\
\hline $\begin{array}{l}\text { Follow Investigator's instruction on diet } \\
\text { and exercise }\end{array}$ & $\mathrm{x}$ & $x$ & $\mathrm{x}$ & $x$ & $\mathrm{x}$ & $x$ & $x$ & $x$ & $x$ & $x$ & $x$ & $x$ & $\mathrm{x}$ & \\
\hline Dispensation of insulin galrgine & & & $x$ & & $\mathrm{x}$ & & & & $x$ & & & $x$ & & \\
\hline Accountability of IMP & & & $\frac{x}{x}$ & & $\mathrm{x}$ & & & & $\mathrm{x}$ & & & $x$ & $\mathrm{x}$ & \\
\hline In In lulin initiation dose & & & $\mathrm{x}$ & $x$ & $\bar{x}$ & $\bar{x}$ & $\bar{x}$ & 8 & $\bar{x}$ & $x$ & $x$ & $\bar{x}$ & $x$ & \\
\hline Self-monitoring of blood glucose & & & & & & & & & & & & & & \\
\hline Dispensation of glucose meter & & $\mathrm{x}$ & & & & & & & & & & & & \\
\hline Dispensation and collection of patient & & $\bar{x}$ & $\mathrm{x}$ & & $\mathrm{X}$ & & & & $x$ & & & $x$ & $\mathrm{X}$ & \\
\hline Iraining of the patient (BG meter and & & $\bar{x}$ & $x$ & & & & & & & & & & & \\
\hline Fraining of the patient (insulin injection) & & & $\mathrm{X}$ & & & & & & & & & & & \\
\hline BG value of 3 consecutive days, at & & & & $X^{*}$ & $\mathrm{X}^{*}$ & $x^{*}$ & $x^{*}$ & $x^{*}$ & $\mathrm{X}^{*}$ & $X^{*}$ & $x^{*}$ & $x^{*}$ & & \\
\hline A 4-points glucose profile (1) & & & $\begin{array}{c}\mathrm{X}^{*} \\
\text { (one day } \\
\text { before } \\
\text { this visit) }\end{array}$ & & & & & & & & & & \begin{tabular}{|c|}
$X^{*}$ \\
$(3$ \\
consecutive \\
days within \\
one week \\
prior to this \\
visit) \\
\end{tabular} & \\
\hline $\begin{array}{l}\text { Other efficacy and safety } \\
\text { DTSQ }\end{array}$ & & X & & & & & & & & & & & $x$ & \\
\hline EQ-5D & & $\frac{x}{x}$ & & & & & & & & & & & $\frac{x}{x}$ & \\
\hline $\begin{array}{l}\text { Hypoglycemia } \\
\text { AEISAE }\end{array}$ & & & & & & $\begin{array}{l}\text { To be ass } \\
\text { To be ass }\end{array}$ & essed thr & Dughout th & $\begin{array}{l}\frac{e \text { whole s }}{e \text { whole s }} \\
\end{array}$ & tudy & & & & \\
\hline
\end{tabular}

Fig. 2 SPIRIT figure. *Sampling before the visit to obtain the result on the day of the visit. (1) Patients will selfmonitor their blood glucose (BG) values immediately before breakfast and $2 \mathrm{~h}$ after breakfast, lunch and dinner. Note: Telephone counselling will be available at any time as required; blood glucose measurements obtained by SMBG will be performed regardless of whether there was a clinical or telephone visit. If treated subjects withdraw from the study prematurely, all final assessment evaluations

HbA1c is assessed at randomization and week 16 post-randomization. Patient-reported outcomes (Diabetes Treatment Satisfaction Questionnaire, DTSQ) and European Quality of Life-5 Dimensions (EQ-5D) are recorded during the run-in period and at week 16 postrandomization.

Adverse events will be collected throughout the study from the signature of the informed consent form to the end of the study as defined (week 16) should be performed at the subject's last visit, except for HbAlc, which should be evaluated only at or after week 12. TC total cholesterol, $H D L-c$ high-density lipoprotein cholesterol, $L D L-c$ low-density lipoprotein cholesterol, $T G$ triglyceride, $F P G$ fasting plasma glucose, $I M P$ investigational medicinal product, $B G$ blood glucose, $F B G$ fasting blood glucose, DTSQ diabetes treatment satisfaction questionnaire, $E Q-5 D$ European quality of life5 dimensions, $A E$ adverse event, $S A E$ serious adverse event

by the protocol for that patient. All adverse events will be managed and reported in compliance with the applicable regulations and included in the final clinical study report.

\section{Sample Size Calculation}

The study sample size was calculated using a formula to test the non-inferiority of the 
difference between two sample proportions [18]. In brief, based on an estimated non-inferiority margin of $10 \%$ (absolute value) and a one-sided alpha of 0.025 with an estimated control group incidence of hypoglycaemia of $32.65 \%$ [19], 440 patients in each group are required to achieve a power of $85 \%$, assuming a $10 \%$ dropout rate.

\section{Statistical Analysis}

Analysis is based on a modified intent-to-treat (ITT) population of all randomized patients who have received at least one dose of insulin glargine and have at least one post-treatment efficacy measurement. Analyses will be repeated in the per protocol population of all patients in the ITT population who have not had a major protocol violation. Safety analyses will be conducted in all patients who are randomized and receive at least one dose of insulin glargine.

For analysis of the primary endpoint, the number and percentage of patients with confirmed or severe hypoglycaemia will be tabulated by treatment arm. Analyses will be performed to obtain a crude estimate of the difference between groups in the percentage of patients with confirmed or severe hypoglycaemia with 95\% CIs for the difference determined using the normal approximation to the binomial. Non-inferiority is established when the confidence interval lies entirely to the left of $10 \%$. For the analysis of secondary endpoints, the percentages of patients achieving HbA1c and FPG targets will be analyzed using the same method as that used for the primary endpoint. Changes in endpoints from baseline to postbaseline visits will be estimated using a mixed model, taking end-of-treatment measurements as the dependent variable, treatment as a fixed effect, baseline measurements as a covariate and patient/visit as a repeated measure indicator. The results will be used to test whether there are any differences in the change from baseline by both treatments, and between the two treatment arms, and 95\% CIs will be estimated. Other continuous variables will be summarized using descriptive statistics.

\section{DISCUSSION}

The Beyond VII study is currently recruiting patients. The results of this study will be clinically important given that diabetes treatment in Asia is currently sub-optimal [11, 20], with delays in the initiation of insulin treatment and subsequent inadequate dose titration, leading to increased levels of diabetic micro- and macrovascular complications. In China, delayed initiation of insulin treatment and inadequate dose titration are also observed and thought to be predominantly due to a lack of medical resources and difficulties in maintaining long-term follow-up of patients [8, 11]. Ineffective blood glucose monitoring is also a barrier to dose titration $[11,20,21]$. Therefore, starting basal insulin treatment at a higher dose may offer a practical solution to this issue as long as it does not lead to a significant increase in adverse events.

The primary objective of the Beyond VII study is therefore to assess whether the safety of commencing basal insulin at a higher dose $(0.3 \mathrm{U} / \mathrm{kg})$ is non-inferior to that of the current standard of care $(0.2 \mathrm{U} / \mathrm{kg})$ in overweight and obese T2DM patients uncontrolled with OADs; this will be based on the percentage of patients with at least one episode of hypoglycaemia (3.9 mmol/1 or severe) during the 16 weeks of treatment.

In treating diabetes, there is a balance between efficacy and safety, namely a trade-off between improved glycaemic control and a risk of hypoglycaemic episodes, and this has resulted in a fear of hypoglycaemia being one of the most common barriers for doctors initiating and titrating basal insulin $[22,23]$. It is therefore important that the potential safety impact of any new treatment paradigm in diabetes is assessed and compared with the current standard of care. To this end, the Beyond VII trial is designed and powered to focus on the safety of a $0.3 \mathrm{U} / \mathrm{kg}$ dose of basal insulin in obese and overweight patients. The safety measurement is the number of patients with at least one episode of hypoglycaemia during treatment, which is both a clinically meaningful assessment and an endpoint that has been used as a safety outcome 
measure in recent clinical trials [24]. Hypoglycaemia is a recognized adverse event of all forms of insulin treatment, although basal insulin is associated with fewer episodes of hypoglycaemia than postprandial insulin treatment $[10,25]$.

In this study, the higher starting dose of $0.3 \mathrm{U} / \mathrm{kg}$ was chosen on the basis of interventional studies conducted in China that reported final doses of insulin glargine of between 0.3 and $0.5 \mathrm{U} / \mathrm{kg}[15,16]$. In addition, the AACE guidelines recommend a higher initial dose of basal insulin between 0.2 and $0.3 \mathrm{U} / \mathrm{kg}$ for patients with HbA1c $>8 \%$ [13]. Franco et al. [14] also support the efficacy and safety of BMIbased higher initial dosing of insulin. In addition, this study has been designed in accordance with the current Consolidated Standards of Reporting Clinical Trials (CONSORT) guidelines and SPIRIT 2013 statement $[26,27]$, which provide guidance on best practice in relation to clinical trial design and reporting.

Considering that commencing insulin glargine treatment at $0.3 \mathrm{U} / \mathrm{kg}$ is not currently a regular clinical practice in the real-world setting, the Beyond VII study is designed as an interventional and "treat-to-target" study that seeks to demonstrate the viability and safety of commencing a patient cohort at a higher $(0.3 \mathrm{U} / \mathrm{kg})$ insulin glargine dose under comparative glycaemic control versus $0.2 \mathrm{U} / \mathrm{kg}$. The average endpoint dose of insulin glargine for Chinese patients in clinical trials is $0.3-0.5 \mathrm{U} / \mathrm{kg}$ $[15,16]$. Based on this fact, we hypothesize that in the real-world setting where a lack of, or inadequate, titration of insulin is common, most patients with an initial dose of $0.3 \mathrm{U} / \mathrm{kg}$ would achieve a better outcome than patients with an initial dose of $0.2 \mathrm{U} / \mathrm{kg}$. The limitation of this trial is that the study population is overweight and obese patients. Current evidence suggests that BMI is an appropriate selection criterion for increasing the starting dose [13]; however, this trial population may be narrower than the real-world population, which could benefit from increased doses of insulin at initiation. Given the limited clinical trial data regarding the use of high-dose insulin, it was considered appropriate to restrict the patient cohort to individuals who are overweight or obese to ensure the safety of the patients; further studies in more generalized populations may be conducted in the future on the basis of the Beyond VII results.

Despite these study design limitations, if the results of Beyond VII demonstrate that a higher starting dose of basal insulin appears efficacious and does not increase the rate of hypoglycaemic events, it may provide a viable treatment option in countries where poor insulin titration has been demonstrated. If a higher starting dose also reduces the need for titration in these patients, there is the potential to maintain better glycaemic control over the longer term and therefore reduce the prevalence of diabetesrelated complications. This, in turn, will help relieve some of the expected future increases in the healthcare burden of diabetes in China.

In summary, the Beyond VII will test the hypothesis that a higher versus standard starting dose of basal insulin has comparable safety to standard dose insulin and can help overcome suboptimal insulin titration in overweight and obese Chinese people with T2DM. The titration of insulin is complex, and the addition of data from a clinical study that reviews the safety of commencing patients on a high-dose insulin regimen will be welcomed by the clinical community. In addition, results from the BEYOND VII study will provide treating clinicians with a framework in which patients on high-dose insulin regimens can be managed.

\section{Trial Status}

This study is currently recruiting participants.

\section{ACKNOWLEDGEMENTS}

We thank all patients and investigators of the Beyond VII Study.

Funding. This study and the article processing charges for this manuscript was sponsored by Sanofi China. All authors had full access to all of the data in this study and take complete responsibility for the integrity of the data and accuracy of the data analysis. 
Medical Writing Assistance. Medical writing services were provided by Hongmei $\mathrm{Xu}$ of Nucleus Global and were funded by Sanofi in accordance with Good Publication Practice (GPP3) guidelines (http://ismpp.org/gpp3).

Authorship. All named authors meet the International Committee of Medical Journal Editors (ICMJE) criteria for authorship for this manuscript, take responsibility for the integrity of the work as a whole and have given final approval to the version to be published.

Author Contributions. Linong Ji contributed to the conception and design of the study, developed the study protocol, will be responsible for the recruitment and the follow-up of the patients enrolled, and will also contribute to the analysis and interpretation of data. Zhengnan Gao, Bimin Shi, Rongwen Bian, Fuzai Yin, and Wuyan Pang contributed to the design of the study and will be responsible for the recruitment and the follow-up of the patients enrolled. Hong Gao contributed to the conception and design of the study and the development of the study protocol. NC contributed to the review and revision of the study protocol. All authors read and approved the final manuscript.

Disclosures. Linong Ji reported receiving consulting and lecture fees from Eli Lilly, Bristol-Myers Squibb, Novartis, Novo Nordisk, Merck, Bayer, Takeda, Sanofi, Roche and Boehringer Ingelheim and research grants from Roche and Sanofi. Hong Gao serves as Medical Manager for Sanofi China. Nan Cui serves as Medical Director for Sanofi China. Zhengnan Gao, Bimin Shi, Rongwen Bian, Fuzai Yin and Wuyan Pang have nothing to disclose.

Compliance with Ethics Guidelines. This non-inferiority trial is designed in accordance with the current Consolidated Standards of Reporting Clinical Trials (CONSORT) guidelines and the Standard Protocol Items for Clinical Trials (SPIRIT) 2013 statement. The study has received ethical approval from the Institutional Review Board (IRB) and Independent Ethics Committee (IEC) of Peking University People's Hospital (Ethics Approval No. 2016PHA006-03) and is conducted in accordance with the Helsinki Declaration of 1964, as revised in 2013. All patients meeting the inclusion criteria will have the study explained to them in detail and will then sign an informed patient consent to participate in the study.

Data Availability. Data sharing is not applicable to this article as no data sets were generated or analyzed during the current study.

Open Access. This article is distributed under the terms of the Creative Commons Attribution-NonCommercial 4.0 International License (http://creativecommons.org/licenses/ by-nc/4.0/), which permits any noncommercial use, distribution, and reproduction in any medium, provided you give appropriate credit to the original author(s) and the source, provide a link to the Creative Commons license, and indicate if changes were made.

\section{REFERENCES}

1. Wang L, Gao P, Zhang M, et al. Prevalence and ethnic pattern of diabetes and prediabetes in China in 2013. JAMA. 2017;317:2515-23.

2. $\mathrm{Xu} \mathrm{Y,} \mathrm{Wang} \mathrm{L,} \mathrm{He} \mathrm{J,} \mathrm{et} \mathrm{al.} \mathrm{Prevalence} \mathrm{and} \mathrm{control} \mathrm{of}$ diabetes in Chinese adults. JAMA. 2013;310:948-59.

3. International Diabetes Federation. Diabetes Atlas, 7th ed. Brussels: International Diabetes Federation, 2015. http://www.diabetesatlas.org. Accessed 21 Sep 2017.

4. Cheng TO. Diabetes epidemic in China and its economic impact. Int J Cardiol. 2011;149:1-3.

5. American Diabetes Association. Standards of medical care in diabetes-2017. Diabetes Care. 2017;40:S1-135.

6. Weng J, Ji L, Jia W, et al. Standards of care for type 2 diabetes in China. Diabetes Metab Res Rev. 2016;32:442-58.

7. Schreiber SA, Ferlinz KandHaak T. The long-term efficacy of insulin glargine plus oral antidiabetic agents in a 32-months observational study of everyday clinical practice. Diabetes Technol Ther. 2008;10:121-7.

8. Ji L, Zhang P, Zhu D, et al. observational registry of basal insulin treatment (ORBIT) in patients with type 2 diabetes uncontrolled with oral 
antihyperglycaemic drugs: real-life use of basal insulin in China. Diabetes Obes Metab. 2017;19:822-30.

9. Riddle MC, Rosenstock J, Gerich J, et al. The treatto-target trial: randomized addition of glargine or human NPH insulin to oral therapy of type 2 diabetic patients. Diabetes Care. 2003;26:3080-6.

10. Ji LN, Lu JM, Guo XH, et al. Glycemic control among patients in China with type 2 diabetes mellitus receiving oral drugs or injectables. BMC Public Health. 2013;13:602.

11. Tsai ST, Pathan F, Ji L, et al. First insulinization with basal insulin in patients with type 2 diabetes in a real-world setting in Asia. J Diabetes. 2011;3:208-16.

12. Odawara $\mathrm{M}$, Ohtani TandKadowaki T. Dosing of insulin glargine to achieve the treatment target in Japanese type 2 diabetes on a basal supported oral therapy regimen in real life: ALOHA study subanalysis. Diabetes Technol Ther. 2012;14:635-43.

13. Garber AJ, Abrahamson MJ, Barzilay JI, et al. Consensus statement by the American Association of clinical endocrinologists and American college of endocrinology on the comprehensive type 2 diabetes management algorithm-2017 executive summary. Endocr Pract. 2017;23:207-38.

14. Franco DR, Baptista J, Abreu FR, et al. Starting glargine in insulin-naive type 2 diabetic patients based on body mass index is safe. World J Diabetes. 2014;5:69-75.

15. Yang $\mathrm{W}, \mathrm{Lv} \mathrm{X}, \mathrm{Li} \mathrm{Q}$, et al. A prospective study to optimize insulin treatment by switching to insulin glargine in type 2 diabetic patients previously uncontrolled on premixed insulin: the optimization study. Curr Med Res Opin. 2012;28:533-41.

16. Zhang B, Zhao J, Yang W, et al. Glycemic control and safety in Chinese patients with type 2 diabetes mellitus who switched from premixed insulin to insulin glargine plus oral antidiabetics: a large, prospective, observational study. Diabetes Metab Res Rev. 2017;33:e2863.

17. Garg SK, Admane K, Freemantle N, et al. Patient-led versus physician-led titration of insulin glargine in patients with uncontrolled type 2 diabetes: a randomized multinational ATLAS study. Endocr Pract. 2015;21:143-57.
18. Chow SC, Shao J, Wang H. Sample size calculations in clinical research, 2nd ed. Chapman Hall/CRC Biostatistics Series; 2008.

19. Owens DR, Traylor L, Dain MP, et al. Efficacy and safety of basal insulin glargine 12 and 24 weeks after initiation in persons with type 2 diabetes: a pooled analysis of data from treatment arms of 15 treat-to-target randomised controlled trials. Diabetes Res Clin Pract. 2014;106:264-74.

20. Kadowaki T, Ohtani T, Naito $Y$, et al. Potential formula for the calculation of starting and incremental insulin glargine doses: ALOHA subanalysis. PLoS One. 2012;7:e41358.

21. Ji L, Newman J, Lu J, et al. Understanding the standard of care in the treatment of type 2 diabetes in China: results from a national survey. Chin Med J (Engl). 2014;127:3524-9.

22. Brod M, Alolga SL, Meneghini L. Barriers to initiating insulin in type 2 diabetes patients: development of a new patient education tool to address myths, misconceptions and clinical realities. Patient. 2014;7:437-50.

23. Sorli CandHeile MK. Identifying and meeting the challenges of insulin therapy in type 2 diabetes. J Multidiscip Healthc. 2014;7:267-82.

24. Balijepalli C, Druyts E, Siliman G, et al. Hypoglycemia: a review of definitions used in clinical trials evaluating antihyperglycemic drugs for diabetes. Clin Epidemiol. 2017;9:291-6.

25. Yki-Jarvinen $H$, Dressler A, Ziemen $M$, et al. Less nocturnal hypoglycemia and better post-dinner glucose control with bedtime insulin glargine compared with bedtime NPH insulin during insulin combination therapy in type 2 diabetes. $\mathrm{HOE}$ 901/3002 Study Group. Diabetes Care. 2000;23:1130-6.

26. Piaggio G, Elbourne DR, Altman DG, et al. Reporting of noninferiority and equivalence randomized trials: an extension of the CONSORT statement. JAMA. 2006;295:1152-60.

27. Chan AW, Tetzlaff JM, Altman DG, et al. SPIRIT 2013 statement: defining standard protocol items for clinical trials. Ann Intern Med. 2013;158:200-7. 